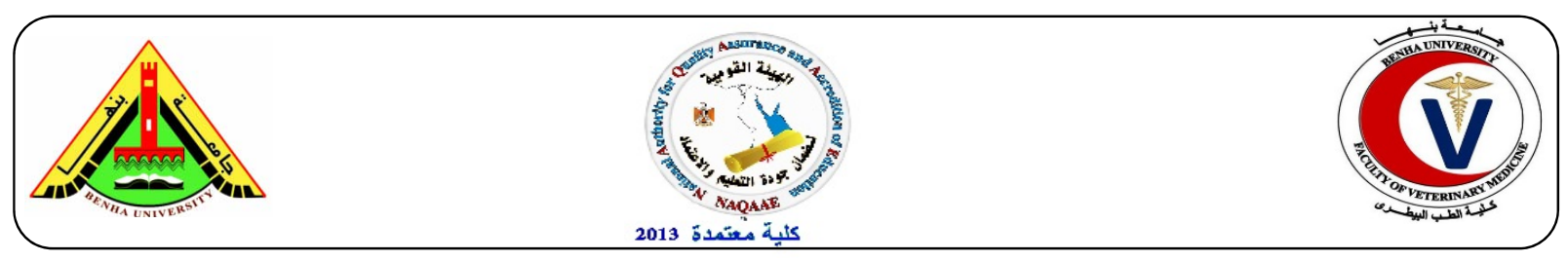

\title{
Efficacy of commercial inactivated Salmonella vaccines in quail
}

\author{
Hala Mahmoud; El-Safty, M. and Hanan, A. Ahmed \\ Central Laboratory for Evaluation of Veterinary Biologics, Abbasia, Cairo Egypt, P.O.B.131
}

\section{A B S T R A C T}

This work aimed to evaluate the efficacy of a commercial inactivated Salmonella vaccine in quails where sixty of three weeks old quails were vaccinated twice via subcutaneous route while another forty birds were kept without vaccination as non-vaccinated control. The immune response of vaccinated birds was estimated by microagglutination test for Salmonella Enteritidis (SE) and Salmonella Typhimurium (ST) antibodies that revealed a detectable increase in antibody titers in vaccinated birds in comparison with the unvaccinated ones. The protection rate of vaccinated quails post challenge with locally isolated Salmonella Enteritidis (SE) and Salmonella Typhimurium (ST) strains were $83.3 \%$ and $90 \%$ respectively. Salmonella was recovered from vaccinated challenged quails at ratio ranged from $23.3 \%-33.3 \%$ for SE and $20 \%-30 \%$ for ST from the heart blood, liver, spleen and caecal junction post challenge. The results of challenge test showed that vaccinated quails were effectively protected against virulent strains of Salmonella Enteritidis (SE) and Salmonella Typhimurium (ST).

Key Words: Salmonella, vaccine, quail.

(http://www.bvmj.bu.edu.eg)

(BVMJ-31(1): 92-95, 2016)

\section{INTRODUCTION}

Salmonella infection is one of the most important bacterial diseases affecting poultry industry especially in intensive systems of rearing. Such infection has a public health importance indicating a need to control Salmonella infection in poultry (Barrow et al., 1999) and any contributions for organism elimination in birds could have a major influence in reduction of its populations under natural conditions (Davies and Breslin, 2004). Control of Salmonellosis in poultry by immunity, whether acquired or innate, is a possible means of containing the problem. Many different serotypes of Salmonella have been isolated from quails, most of them have a public health significance, but some include S. Typhimurium, $S$. Enteritidis and S. gallinarum that could cause considerable losses in quails of less than a few weeks of age (Sander et al., 2001). Widespread usage of antibiotics has led to the emergence of multiple antibiotic-resistant bacteria (Gast et al., $2004 \mathrm{a}, \mathrm{b}$ ). Control of Salmonellosis in poultry is posing itself as one of the difficult problems not only for those who are concerned with poultry industry, but also for public health hazard because of the fact that most of the serovars of Salmonella which poultry harbour can act as potential pathogens for human, this problem has indicated an increasing requirement for effective vaccines to control this important zoonotic infection (Methner et al., 2006 and Barrow, 2007). Both live attenuated and inactivated vaccines are available where live vaccines induce better protection than inactivated ones while inactivated vaccines appeal more to producers and regulators because they do not pose the possible public health risk that accompany the use of live vaccines (Barrow, 2007).

The objective of the present work is to evaluate the efficacy of the commercial inactivated Salmonella vaccine in immunizing and protecting quails against experimental Salmonella infection.

\section{MATERIAL AND METHODS}

\subsection{Avian Salmonellosis vaccine:}

Commercial oil inactivated Salmonella vaccine prepared from S. Typhimurium and S. Enteritidis strains was used for vaccination of quails.

\subsection{Salmonella strains:}

Local isolates of $S$. Typhimurium and $S$. Enteritidis isolated from infected poultry were obtained from Veterinary Serum and Vaccine Research Institute (VSVRI), Abbasia, Cairo, Egypt. These isolates were used for experimental challenge of vaccinated quails. 


\subsection{Quails:}

One hundred, three weeks old quails were obtained from the farm of Faculty of Agriculture, Cairo university, Egypt. These quails were used to evaluate the potency of inactivated Salmonella vaccine. These birds were tested and found to be free from Salmonella infection and antibodies as determined serologically. All birds were housed under hygienic measures in separate isolates receiving balanced ration and adequate water.

\subsection{Mice:}

A total of 250 Swiss Albino mice of about 1520 g body weight supplied by Veterinary Serum and Vaccine Research Institute, were used for determination of $\mathrm{LD}_{50}$ of $\mathrm{S}$. Typhimurium and $\mathrm{S}$. Enteritidis strains.

\subsection{Potency test:}

One hundred quails at 3 weeks old were divided as follow: 60 quails were vaccinated with the vaccine with 2 doses each of $0.5 \mathrm{ml}$ inoculated subcutaneously in the dorsal aspect of the neck with 3 weeks interval depending on the recommended dose according to manufacturer instructions. 40 quails were kept without vaccination as control. All birds were housed in separate isolates under hygienic measures receiving adequate ration and water. Serum samples were obtained weekly from all birds to follow up the induced antibody levels.

\subsection{Challenge test:}

Vaccinated and non-vaccinated control quails groups (each group was divided into two subgroups) were challenged two weeks post the $2^{\text {nd }}$ dose with the virulent S. Typhimurium and $\mathrm{S}$. Enteritidis strains using $0.1 \mathrm{ml}$ of $10^{8}$ colony forming units $/ \mathrm{ml}$ inoculated intramuscularly (Adriaesen, et al., 2007).

2.7. Serological evaluation of humeral immune response of the vaccinated quails by Microagglutination test (MAT):

This test was carried out to estimate Salmonella antibodies in vaccinated birds as described by Thaxton et al. (1970) and Brown et al. (1981). The geometric mean ST antibody titers were calculated according to Brugh (1977).

\subsection{Recovery of Salmonella strains from challenged quails:}

On the $4^{\text {th }}$ week post challenge, samples were collected from the heart blood, liver, spleen and caecal junction from vaccinated and nonvaccinated challenged quails for recovery of the organism.

\section{RESULTS}

During the present work, a commercial oil inactivated Salmonella vaccine was successfully used to vaccinate quails against Salmonella Enteritidis and S. Typhimurium infections, where the humoral immune response was evaluated in vaccinated quails by microagglutination test as demonstrated in (Table 1). The Geometric mean of Salmonella antibody titers against SE and ST strains in sera of vaccinated birds were detectably increased from [6] and [7] pre-vaccination to reach [343] and [422], respectively at the $5^{\text {th }}$ week post vaccination with inactivated Salmonella vaccine, while the control unvaccinated birds showed steady levels [6-9].

Concerning the protection efficacy of Salmonella vaccine, (Table 2) showed that the protection rate was $83.3 \%$ and $90 \%$ in vaccinated quails when challenged with virulent SE and ST strains, respectively, while the control unvaccinated group was unable to withstand the experimental infection with virulent SE and ST strains confirming that the vaccine was effectively potent and hence able to protect quails against infection. On the other hand, (Table 3) showed that the Salmonella Enteritidis and S. Typhimurium organism could be recovered from vaccinated challenged quails with commercial inactivated Salmonella vaccine at ratio from $33.3 \%-23.3 \%$ for SE and 20\% - 30\% for ST from heart blood, liver, spleen and caecal junction on the $4^{\text {th }}$ week post challenge, while these ratios were $75-90 \%$ from control unvaccinated birds.

\section{DISCUSSION}

Salmonella infections in poultry are probably the most important source of Salmonellaassociated food-poisoning in human and the contribution of different species to human infection bears some relationship to the quantity of meat from each species that is consumed. Consumption of poultry meat is much greater in some countries where the incidence of human infection was originated from this source (Barrow, 2007). Incidence of human infection arising from consumption of duck meat is likely to be much greater as it is with human infection arising from chickens (Methner et al., 2006). Accordingly, the present study aimed to answer the question about to any extent quails could be protected against Salmonella infection? And parallel to this respect how aid to minimize Salmonella infection in man? During the present work, a commercial oil inactivated Salmonella vaccine was successfully used to vaccinate quails against Salmonella 
Table (1): Salmonella Enteritidis (SE) and Typhimurium (ST) antibody titers in quails sera as measured by microagglutination test

\begin{tabular}{|c|c|c|c|c|c|c|c|}
\hline \multirow[t]{2}{*}{ Quails Groups } & \multirow[t]{2}{*}{ Strain used } & \multicolumn{6}{|c|}{$\begin{array}{l}\text { Geometric mean of Salmonella antibody titers } \\
\text { on periods post vaccination }\end{array}$} \\
\hline & & Prevacc. & $1 \mathrm{WPV}$ & $2 \mathrm{WPV}$ & $3 \mathrm{WPV}$ & $4 \mathrm{WPV}$ & $5 \mathrm{WPV}$ \\
\hline \multirow{2}{*}{ Vaccinated } & SE & 6 & 28 & 57 & 75 & 226 & 343 \\
\hline & ST & 7 & 43 & 70 & 92 & 299 & 422 \\
\hline \multirow{2}{*}{ Control } & $\mathrm{SE}$ & 8 & 7 & 8 & 7 & 8 & 9 \\
\hline & ST & 6 & 7 & 6 & 8 & 6 & 7 \\
\hline
\end{tabular}

Table (2): Protective efficacy of inactivated Salmonella vaccine against challenge with virulent Salmonella Enteritidis (SE) and S. Typhimurium (ST) strains

\begin{tabular}{ccccc}
\hline $\begin{array}{c}\text { Quail } \\
\text { Groups }\end{array}$ & $\begin{array}{c}\text { Challenge } \\
\text { strain }\end{array}$ & $\begin{array}{c}\text { No. } \\
\text { of challenged quails }\end{array}$ & $\begin{array}{c}\text { No. } \\
\text { of survived quails }\end{array}$ & $\begin{array}{c}\text { Protection } \\
\text { Rate }\end{array}$ \\
\hline Vaccinated & SE & 30 & 25 & $83.3 \%$ \\
& ST & 30 & 27 & $90 \%$ \\
Control & SE & 20 & 9 & $45 \%$ \\
& ST & 20 & 8 & $40 \%$ \\
\hline
\end{tabular}

Table (3): Recovery of Salmonella strains from challenged quails

\begin{tabular}{cccccc}
\hline \multirow{2}{*}{ Quail Groups } & Challenge strain & \multicolumn{4}{c}{ Number of positive samples for Salmonella recovery } \\
& & Heart blood & Liver & Spleen & Caecal \\
junction
\end{tabular}

Enteritidis and S. Typhimurium infections. The Geometric mean of Salmonella antibody titers against SE and ST strains in sera of vaccinated birds detectably increased at $5^{\text {th }}$ week post vaccination while the control unvaccinated birds showed steady levels.

Concerning the protection efficacy of Salmonella vaccine, the protection rate was satisfactory potent in vaccinated quails when challenged with virulent SE and ST strains, while the control unvaccinated group was unable to withstand the experimental infection with virulent SE and ST strains confirming that the vaccine was effectively potent and hence able to protect quails against infection. These results came in agreement with previous report ((Uytteroek et al., 1989; Nakamura et al., 1994) and Barrow, (2007) who recommended the use of formalized inactivated oil emulsion Salmonella vaccine for protection of poultry against infection. On the other hand, the Salmonella Enteritidis and S. Typhimurium organism could be re-isolated from vaccinated challenged quails with commercial inactivated Salmonella vaccine from heart blood, liver, spleen and caecal junction on the $4^{\text {th }}$ week post challenge by a lower ratio than from control unvaccinated birds

These results agreed with that Salmonella vaccine protects against experimental challenge with shedding of the organism on the same period with declined rate post challenge with indication that the highest incidence of the organism is that in the caecal junction ((Uytteroek et al., 1989 and Timms et al., 1990) 
So, it could be concluded that the vaccination studies performed here showed that quails immunized with two doses of inactivated Salmonella vaccine were protected to a high degree against challenge with the same pathogenic Salmonella strains.

\section{REFERENCES}

Barrow, P.A., Lovell, M.A., Murphy, C.K., Page, K. 1999. Salmonella infection in commercial line of ducks; experimental studies on virulence; intestinal colonization and immune protection. Epidem. Infect., 123, 121-132.

Davies, R.H., Breslin, M. 2004. Observations on Salmonella contamination of commercial laying farms before and after cleaning and disinfection. Vet. Rec. 152, 283-287.

Sander, J., Hudson, C.R., Dufour-Zavala, L., Waltman, W.D., Lobsinger, C., Thayer S.G., Otalora, R., Maurer, J.J. 2001. Dynamics of Salmonella contamination in a commercial quail operation. Avian Dis. 45(4), 1044-9.

Gast, R.K., Mitchell, B.W., Holt, P.S. 2004a. Detection of airborne Salmonella Enteritidis in the environment of experimentally infected laying hens by an electrostatic sampling device. Avian Dis. 48,145-154.

Gast, R.K., Mitchell, B.W., Holt, P.S. 2004b. Evaluation of culture media for detecting airborne Salmonella Enteritidis collected with an electrostatic sampling device from the environment of experimentally infected laying hens. Poult. Sci. 83, 1106-1111.
Methner, U., Reiche, R., Bohland, K. 2006. Occurrence of Salmonella in laying hens in different housing systems in control. Avian Dis., 46: 453-461.

Barrow, P.A. 2007. Salmonella infections: immune and non-immune protection with vaccines. Avian Path. 36 (1), 1-13.

Adriaesen, C., Henride, G., Jean, Q., Tian, S.; Elne, G. 2007. Salmonella Enteritidis vaccine allows serological differentiation between vaccinated and infected animals. Infect. Immun., 44, 134-145.

Thaxton, P.; Williams, J.E.; Siegal, H.S. 1970. Microtitration of Salmonella_Pullorum agglutinins. Avian Dis., 14, $813-816$.

Brown, S.L.; Klin, F.T.; Jones, W.L. 1981. Safranin "O" stained antigen microagglutination test for detection of brucella antibodies. J. Clin. Microbiol., 13, $398-400$.

Brugh, M.J. 1977. A simple method for recording and analyzing serological data Avian Dis., 22, 362-366.

Uytteroek, E., Devriese, L.A., Gevaert, D. 1989. Effect of different chemotherapeutics on experimental salmonellosis in pigeons. Vlaams Diergeneeskundig tijidschrift, 58, 51-54.

Nakamura, M., Nagamine, N., Norimatsu, M., Suzuki, S., Ohishi, K., Kijima, M., Tamura, Y., Sato, S. 1994. The ability of Salmonella Enteritidis isolated from chicks imported from England to cause trans-ovarian infection. J. Vet. Med. Sci., 55(1), 135 - 136.

Timms, L.M.; Marshal, R.N.; Breslin, M.F. 1990. Laboratory assessment of protection given by Salmonella Enteritidis PT4 inactivated, adjuvented vaccine. Vet. Rec., 127, 22 - 29. 